\title{
Study on The Symmetry and Conserved Quantities for Hamilton Systems
}

\author{
Shu-long Gu \\ School of Physics and Electronic Engineering, Nanjing \\ Xiaozhuang University \\ Nanjing, China \\ e-mail: gs12142@sohu.com
}

\author{
Kai-xia Wei \\ School of Physics and Electronic Engineering, Nanjing \\ Xiaozhuang University \\ Nanjing, China \\ e-mail:wkxwjm@163.com
}

$$
\begin{gathered}
\dot{q}_{s}=\frac{\partial H}{\partial p_{s}} \\
\dot{p}_{s}=\frac{\partial H}{\partial q_{s}}
\end{gathered}
$$

Where is the Hamilton.introduce the infinitesimal transformations with respect to time, generalized coordinates and generalized momentums

$$
\begin{aligned}
& t^{*}=t+\Delta t \\
& q_{s}^{*}\left(t^{*}\right)=q_{s}(t)+\Delta q_{s} \\
& p_{s}^{*}\left(t^{*}\right)=p_{s}(t)+\Delta p_{s}
\end{aligned}
$$

Or their expansion formula

$$
\begin{gathered}
t^{*}=t+\varepsilon \tau(t, \mathbf{q}, \mathbf{p}) \\
q_{s}^{*}\left(t^{*}\right)=q_{s}(t)+\varepsilon \xi_{s}(t, \mathbf{q}, \mathbf{p}) \\
p_{s}^{*}\left(t^{*}\right)=p_{s}(t)+\varepsilon \eta_{s}(t, \mathbf{q}, \mathbf{p})
\end{gathered}
$$

Where $\varepsilon$ is an infinitesimal parameter, $\tau, \xi_{s}$ and $\eta_{s}$ are called infinitesimal generators. Under the infinitesimal transformation (5), Hamilton $H(t, \mathbf{q}, \mathbf{p})$ becomes $H\left(t^{*}, \mathbf{q}^{*}, \mathbf{p}^{*}\right)$.

Definition 1: Under the infinitesimal transformation (5), if the canonical (3) keep their form invariant, much attention ${ }^{[5-10]}$. In this paper, we study Hojm conserved quantities by using the Mei form invariance for the Hamilton systems.

\section{DEFINITION AND CRITERION OF FORM INVARIANCE FOR HAMILTON SYSTEMS}

If the differential equations of motion of a mechanical system can be written in the form

$$
\frac{d}{d t} \frac{\partial L}{\partial \dot{q}_{s}}-\frac{\partial L}{\partial q_{s}}=0
$$

Then such system is called a lagrangian system. Introducing the generalized momentums and the Hamilton

$$
\begin{gathered}
P_{s}=\frac{\partial L}{\partial q_{s}} \\
H=\sum p_{s} \dot{q}_{s}-L
\end{gathered}
$$

Then (1) may be written in the canonical form

$$
\begin{gathered}
\dot{q}_{s}=\frac{\partial H^{*}}{\partial p_{s}} \\
\dot{p}_{s}=\frac{\partial H^{*}}{\partial q_{s}}
\end{gathered}
$$

Where

$$
H^{*}=H\left(t^{*}, \mathbf{q}^{*}, \mathbf{p}^{*}\right)
$$

Then such invariance is called a form invariance of the Hamilton systems. Introduce the differential operator of the infinitesimal generators

$$
X^{(0)}=\tau \frac{\partial}{\partial t}+\xi_{k} \frac{\partial}{\partial q_{k}}+\eta_{k} \frac{\partial}{\partial p_{k}}
$$


$X^{(1)}=X^{(0)}+\left(\dot{\xi}_{k}-\dot{q}_{k} \dot{\tau}\right) \frac{\partial}{\partial \dot{q}_{k}}+\left(\dot{\eta}_{k}+\dot{p}_{k} \dot{\tau}_{k}\right) \frac{\partial}{\partial \dot{p}_{k}}$

Expanding $H^{*}$, one has

$$
H^{*}=H(t, \mathbf{q}, \mathbf{p})+\varepsilon\left[X^{(0)}(H)\right]+O\left(\varepsilon^{2}\right)
$$

From (6) - (10), the following criterion can be obtained.

Criterion 1: the infinitesimal transformation (5) is a Mei symmetric transformation of the system(3), if and only if the infinitesimal generators $\tau, \xi_{s}$ and $\eta_{s}$ satisfy the following conditions

$$
\begin{aligned}
& \frac{\partial X^{(0)}(H)}{\partial p_{s}}=0 \\
& \frac{\partial X^{(0)}(H)}{\partial q_{s}}=0
\end{aligned}
$$

Proof: substituting (10) into (6), using (3), and neglecting the higher infinitesimal terms, (11) will be obtained.

\section{THE HOJMAN CONSERVED QUANTITY FOR HAMILTON SYSTEMS}

The basic idea of the Lie symmetry is to keep the equations of motion(3) invariant under the infinitesimal transformations (5). For convenience, equation(3) are rewritten in following form

$$
\begin{gathered}
\dot{q}_{s}=g_{s}(t, \mathbf{q}, \mathbf{p}) \\
\dot{p}_{s}=h_{s}(t, \mathbf{q}, \mathbf{p})
\end{gathered}
$$

Where $g_{s}=\partial H / \partial p_{s}$, and $h_{s}=-\partial H / \partial \dot{q}_{s}$.

Definition 2: the infinitesimal transformations (5) is a Lie,s symmetric transformation of the system(3), if and only if there exist function $\tau, \xi_{s}$ and $\eta_{s}$ that satisfy the following determining equations

$$
\begin{gathered}
\frac{\bar{d} \xi_{s}}{d t}-g_{s} \frac{\bar{d} \tau}{d t}=\tau \frac{\partial g_{s}}{\partial t}+\xi_{k} \frac{\partial g_{s}}{\partial q_{k}}+\eta_{k} \frac{\partial g_{s}}{\partial p_{k}} \\
(s, k=1,2 \cdots, n) \\
\frac{\bar{d} \eta_{s}}{d t}-h_{s} \frac{\bar{d} \tau}{d t}=\tau \frac{\partial h_{s}}{\partial t}+\xi_{k} \frac{\partial g_{h s}}{\partial q_{k}}+\eta_{k} \frac{\partial h_{s}}{\partial p_{k}} \\
(s, k=1,2 \cdots, n)
\end{gathered}
$$

Where

$$
\begin{gathered}
\frac{\bar{d}}{d t}=\frac{\partial}{\partial t}+g_{s} \frac{\partial}{d q_{s}}+h_{s} \frac{\partial}{\partial p_{s}} \\
(s=1,2 \cdots, n)
\end{gathered}
$$

In terms of the generators of the Lie symmetry for e(3), the following theorem concerning conserved quantities can be proved.

Theorem 1: the system(3) possesses the following conserved quantity

$$
I=\frac{1}{\mu} \frac{\partial(\mu \tau)}{\partial t}+\frac{1}{\mu} \frac{\partial\left(\mu \xi_{s}\right)}{\partial q_{s}}+\frac{1}{\mu} \frac{\partial\left(\mu \eta_{s}\right)}{\partial p_{s}}-\frac{\bar{d}}{d t} \tau
$$

If the infinitesimal the generators $\tau, \xi_{s}$ and $\eta_{s}$ satisfy the following determining (13), and the $\mu(t, \mathbf{q}, \mathbf{p})$ admits the following equation

$$
\frac{\bar{d}}{d t} \ln \mu=-\tau \frac{\partial g_{s}}{d q_{s}}-\frac{\partial h_{s}}{\partial p_{s}}
$$

Proof: from (15), one has

$$
\begin{gathered}
\frac{\bar{d} I}{d t}=\frac{\bar{d}}{d t}\left(\frac{1}{\mu} \frac{\partial \mu}{\partial t}\right)+\frac{\bar{d}}{d t} \frac{\partial \tau}{\partial t}+\frac{\bar{d}}{d t}\left(\frac{1}{\mu} \frac{\partial \mu}{\partial q_{s}} \xi_{s}\right) \\
+\frac{\bar{d}}{d t} \frac{\partial \xi_{s}}{\partial q_{s}}+\frac{\bar{d}}{d t}\left(\frac{1}{\mu} \frac{\partial \mu}{\partial \eta_{s}}\right)+\frac{\bar{d}}{d t} \frac{\partial \eta_{s}}{\partial p_{s}}-\frac{\bar{d}}{d t} \frac{\partial \tau}{\partial t} \\
(s=1,2 \cdots, n)
\end{gathered}
$$

It is straightforward to show that for any $A(t, \mathbf{q}, \mathbf{p})$

$$
\begin{aligned}
\frac{\bar{d}}{d t} \frac{\partial \tau}{\partial t}= & \frac{\partial}{\partial t} \frac{\bar{d} \tau}{d t}-\frac{\partial g_{s}}{\partial t} \frac{\partial \tau}{\partial q_{s}}-\frac{\partial h_{s}}{\partial t} \frac{\partial \tau}{\partial p_{s}} \\
& (s=1,2 \cdots, n) \quad(18 \alpha) \\
\frac{\bar{d}}{d t} \frac{\partial \xi_{s}}{\partial q_{s}}= & \frac{\partial}{\partial q_{s}} \frac{\bar{d} \xi_{s}}{d t}-\frac{\partial g_{s}}{\partial q_{k}} \frac{\partial \xi_{k}}{\partial q_{s}}-\frac{\partial h_{s}}{\partial q_{k}} \frac{\partial \xi_{k}}{\partial p_{s}} \\
& (s=1,2 \cdots, n) \quad(18 \beta) \\
\frac{\bar{d}}{d t} \frac{\partial \eta_{s}}{\partial q_{s}}= & \frac{\partial}{\partial p_{s}} \frac{\bar{d} \eta_{s}}{d t}-\frac{\partial g_{s}}{\partial p_{k}} \frac{\partial \eta_{k}}{\partial q_{s}}-\frac{\partial h_{s}}{\partial p_{k}} \frac{\partial \eta_{k}}{\partial p_{s}} \\
& (s=1,2 \cdots, n) \quad(18 \chi)
\end{aligned}
$$

And substituting (18) into (17) and using (13),we obtain

$$
\begin{gathered}
\frac{\bar{d} I}{d t}=\frac{\bar{d}}{d t}\left(\frac{1}{\mu} \frac{\partial \mu}{\partial t} \tau\right)+\frac{\bar{d}}{d t}\left(\frac{1}{\mu} \frac{\partial \mu}{\partial q_{s}} \xi_{s}\right)+\frac{\bar{d}}{d t}\left(\frac{1}{\mu} \frac{\partial \mu}{\partial p_{s}} \eta_{s}\right)+\left(\frac{\partial g_{s}}{\partial q_{s}}+\frac{\partial h_{s}}{\partial p_{s}}\right) \frac{\bar{d} \tau}{d t} \\
+\frac{\partial}{\partial t}\left(\frac{\partial g_{s}}{\partial q_{s}}+\frac{\partial h_{s}}{\partial p_{s}}\right) \tau+\frac{\partial}{\partial q_{k}}\left(\frac{\partial g_{s}}{\partial q_{s}}+\frac{\partial h_{s}}{\partial p_{s}}\right) \xi_{k}+\frac{\partial}{\partial q_{k}}\left(\frac{\partial g_{s}}{\partial q_{s}}+\frac{\partial h_{s}}{\partial p_{s}}\right) \eta_{k} \\
(s=1,2 \cdots, n)
\end{gathered}
$$

Finding the partial differential of (16) with respect to $t, q_{k}$ and $p_{k}$ respectively, and substituting the results into (19), and using (13),one can get

$$
\frac{\bar{d} I}{d t}=0
$$

By virtue of above theorem, one can easily deduce following corollaries:

Corollary 1: the system (3) possesses the following conserved quantity

$$
I=\frac{1}{\mu} \frac{\partial\left(\mu \xi_{s}\right)}{\partial q_{s}}+\frac{1}{\mu} \frac{\partial\left(\mu \eta_{s}\right)}{\partial p_{s}} \quad(s=1,2 \cdots, n)
$$

If the infinitesimal generators $\tau, \xi_{s}$ and $\eta_{s}$ satisfy

$\frac{\bar{d} \xi_{s}}{d t}=\frac{\partial g_{s}}{\partial q_{k}} \xi_{k}+\frac{\partial g_{s}}{\partial p_{k}} \eta_{k}(s, k=1,2 \cdots, n)$ 


$$
\begin{aligned}
& \frac{\bar{d} \eta_{s}}{d t}=\frac{\partial h_{s}}{\partial q_{k}} \xi_{k}+\frac{\partial h_{s}}{\partial p_{k}} \eta_{k}(s, k=1,2 \cdots, n) \\
& \text { And } \mu(t, \mathbf{q}, \mathbf{p}) \text { admits the (16). }
\end{aligned}
$$

Corollary 2: the system (4) possesses the following conserved quantity

$I=\frac{1}{\mu} \frac{\partial(\mu \tau)}{\partial t}+\frac{1}{\mu} \frac{\partial\left(\mu \eta_{s}\right)}{\partial p_{s}}-\frac{\bar{d} \tau}{d t}(s=1,2 \cdots, n)$

If the infinitesimal generators $\tau, \xi_{s}$ and $\eta_{s}$ satisfy

$$
\begin{gathered}
g_{s} \frac{\bar{d} \tau}{d t}+\tau \frac{\partial g_{s}}{\partial t}+\eta_{k} \frac{\partial g_{s}}{\partial p_{k}}=0(s, k=1,2 \cdots, n) \\
\frac{\bar{d} \eta_{s}}{d t}-h_{s} \frac{\bar{d} \tau}{d t}-\tau \frac{\partial h_{s}}{\partial t}-\eta_{k} \frac{\partial h_{s}}{\partial p_{k}}=0
\end{gathered}
$$

And function $\mu(t, \mathbf{q}, \mathbf{p})$ admits the (16).

Corollary 3: the system (4) possesses the following conserved quantity

$$
I=\frac{1}{\mu} \frac{\partial(\mu \tau)}{\partial t}+\frac{1}{\mu} \frac{\partial\left(\mu \xi_{s}\right)}{\partial q_{s}}-\frac{\bar{d} \tau}{d t}(s=1,2 \cdots, n)
$$

If the infinitesimal generators $\tau, \xi_{s}$ and $\eta_{s}$ satisfy

$$
\begin{aligned}
& h_{s} \frac{\bar{d} \tau}{d t}+\tau \frac{\partial h_{s}}{\partial t}+\eta_{k} \frac{\partial h_{s}}{\partial p_{k}}=0(s, k=1,2 \cdots, n) \\
& \frac{\bar{d} \xi_{s}}{d t}-g_{s} \frac{\bar{d} \tau}{d t}-\tau \frac{\partial g_{s}}{\partial t}-\xi_{k} \frac{\partial g_{s}}{\partial p_{k}}=0
\end{aligned}
$$$$
\text { And function } \mu(t, \mathbf{q}, \mathbf{p}) \text { admits (16). }
$$

\section{NECESSARY AND SUFFICIENT CONDITION UNDER WITCH THE FORM INVARIANCE IS A LIE SYMMETRY}

From the deductions of (11) and (13), it can be seen that the form invariance is generally different from the Lie symmetry. For seeking their relations, the equation (3) may be rewritten as follows:

$$
\begin{aligned}
& F(t, \mathbf{q}, \mathbf{p}, \dot{\mathbf{q}})=\dot{q}_{s} \frac{\partial H}{\partial p_{s}}=0 \\
& G(t, \mathbf{q}, \mathbf{p}, \dot{\mathbf{q}})=\dot{p}_{s} \frac{\partial H}{\partial p_{s}}=0
\end{aligned}
$$

Then for the system (3), the determining equations of a Lie,s symmetry have new form

$$
\begin{aligned}
& X^{(1)}(F)=0 \\
& X^{(1)}(G)=0
\end{aligned}
$$

Some direction calculations yield

$$
\begin{aligned}
& \frac{\partial X^{(0)}(H)}{\partial p_{s}}=\dot{\xi}_{s}-\dot{q}_{s} \dot{\tau}-X^{(1)}(F) \\
& +\frac{\partial \tau}{\partial p_{s}} \frac{\partial H}{\partial t}+\frac{\partial \xi_{k}}{\partial p_{s}} \frac{\partial H}{\partial q_{k}}+\frac{\partial \eta_{k}}{\partial p_{s}} \frac{\partial H}{\partial p_{k}}
\end{aligned}
$$

$$
\begin{aligned}
& \frac{\partial X^{(0)}(H)}{\partial q_{s}}=-\dot{\eta}_{s}-\dot{p}_{s} \dot{\tau}+X^{(1)}(G) \\
& +\frac{\partial \tau}{\partial q_{s}} \frac{\partial H}{\partial t}+\frac{\partial \xi_{k}}{\partial q_{s}} \frac{\partial H}{\partial q_{k}}+\frac{\partial \eta_{k}}{\partial q_{s}} \frac{\partial H}{\partial p_{k}}
\end{aligned}
$$

Equation(29) demonstrates the relation between the form invariance and the Lie symmetry. From the relation, the following proposition can be derived.

Proposition 1: for the Hamilton system, the necessary and sufficient condition under which the form invariance is a Lie symmetry is that the following relations hold

$$
\begin{aligned}
& \dot{\xi}_{s}-\dot{q}_{s} \dot{\tau}-\frac{\partial \tau}{\partial p_{s}} \frac{\partial H}{\partial t}+\frac{\partial \xi_{k}}{\partial p_{s}} \frac{\partial H}{\partial q_{k}}+\frac{\partial \eta_{k}}{\partial p_{s}} \frac{\partial H}{\partial p_{k}}=0 \\
& \dot{\eta}_{s}-\dot{p}_{s} \dot{\tau}-\frac{\partial \tau}{\partial q_{s}} \frac{\partial H}{\partial t}-\frac{\partial \xi_{k}}{\partial q_{s}} \frac{\partial H}{\partial q_{k}}-\frac{\partial \eta_{k}}{\partial q_{s}} \frac{\partial H}{\partial p_{k}}=0
\end{aligned}
$$

Let the sum of the coefficient of the terms which dependent on or respectively equals zero, and the sum of the remainder of terms equals also zero, one has

$$
\begin{aligned}
& \dot{\tau}=0 \\
& \dot{\xi}_{s}+\frac{\partial \tau_{0}}{\partial p_{s}} \frac{\partial H}{\partial t}+\frac{\partial \xi_{k}}{\partial p_{s}} \frac{\partial H}{\partial q_{k}}+\frac{\partial \eta_{k}}{\partial p_{s}} \frac{\partial H}{\partial p_{k}}=0 \\
& \dot{\eta}_{s}-\frac{\partial \tau_{0}}{\partial q_{s}} \frac{\partial H}{\partial t}-\frac{\partial \xi_{k}}{\partial q_{s}} \frac{\partial H}{\partial q_{k}}-\frac{\partial \eta_{k}}{\partial q_{s}} \frac{\partial H}{\partial p_{k}}=0
\end{aligned}
$$

Proof: substitution of (11) and (30) into (29) leads to $X^{(1)}(F)=0$ and $X^{(1)}(G)=0$. According to the determining (28), we know that the form invariance is a Lie symmetry.

Particularly, if $\tau=0$, then the conditions (30) become

$$
\begin{aligned}
& \dot{\xi}_{s}+\frac{\partial \xi_{k}}{\partial p_{s}} \frac{\partial H}{\partial q_{k}}+\frac{\partial \eta_{k}}{\partial p_{s}} \frac{\partial H}{\partial p_{k}}=0 \\
& \dot{\eta}_{s}-\frac{\partial \xi_{k}}{\partial q_{s}} \frac{\partial H}{\partial q_{k}}-\frac{\partial \eta_{k}}{\partial q_{s}} \frac{\partial H}{\partial p_{k}}=0
\end{aligned}
$$

\section{HOJMAN CONSERVED QUANTITY DEDUCED FROM FORM INVARIANCE}

The Hojman conserved quantity can be located by using the form invariance.

Proposition 2: For the Hamilton system, under the infinitesimal transformation (5), if the infinitesimal generators $\tau, \xi_{s}$ and $\eta_{s}$ satisfy (11) and (30), and there exists a function $\mu=\mu(t, \mathbf{q}, \mathbf{p})$ admits the equation (16), then form invariance leads to the Hojman conserved quantity(15).

Proof: if the infinitesimal generators $\tau, \xi_{s}$ and $\eta_{s}$ satisfy (11) and (30), by using proposition 1 , we now that the generators are also Lie symmetry. And we can subsequently obtain the conserved quantity (15) by using the theorem 1. 
Proposition 3: For the Hamilton system, under the infinitesimal transformation(5), if the infinitesimal generators $\tau, \xi_{s}$ and $\eta_{s}$ satisfy (11) and (32), and there exists a function $\mu=\mu(t, \mathbf{q}, \mathbf{p})$ admits the (16), then form invariance leads to the Hojman conserved quantity (21).

Proof: if the infinitesimal generators $\tau, \xi_{s}$ and $\eta_{s}$ satisfy (11) and (32), proposition 1 means that the generators are also Lie symmetrical. Corollary 1 yields subsequently the conserved quantity (21).

\section{AN ILLUSTRATIVE EXAMPLE}

As an illustration of the theory developed in the preceding sections, consider the case of a simple degree of freedom linear damped oscillator

$$
\ddot{q}+\gamma \dot{q}=0
$$

First, transform Eq.(33) into a Hamilton system, and its Lagrangian is

$$
L=\frac{1}{2} e^{\lambda t} \dot{q}^{2}
$$

Therefore

$$
\begin{aligned}
p & =\frac{\partial L}{\partial \dot{q}}=e^{\gamma t} \dot{q}^{2} \\
H & =p \dot{q}-L=\frac{1}{2} e^{-\gamma t} p^{2}
\end{aligned}
$$

Eq.(11) leads to

$$
\begin{aligned}
& \frac{\partial \tau}{\partial q}\left(-\frac{1}{2} \not p\right)+\frac{\partial \eta}{\partial q}=0 \\
& \text { It can be easily verified that } \\
& \begin{array}{l}
\tau=0, \quad \xi=1, \quad \eta=0 ; \\
\tau=1, \quad \xi=q, \quad \eta=\frac{1}{2} \not p
\end{array}
\end{aligned}
$$$$
\frac{\partial \tau}{\partial p}\left(-\frac{1}{2} \not p^{2}\right)-\not p \tau+\frac{\partial \eta}{\partial p} p+\eta=0
$$

Are toe solution sets of (36). Since the generator (37) satisfies the (13), so it is also the Lie symmetry of the system (34). However, the generators (38) do not satisfy the (13), so it is not the Lie symmetry of the system (34). From (16), one has

$$
\frac{\bar{d} \tau}{d t} \ln \mu=0
$$

Equation (39) exists a solution

$$
\mu=e^{-\gamma t} p+\not p
$$

Inserting (37) and (40) into (25) leads to conserved quantity

$$
I=\frac{\gamma}{e^{-\gamma t} p+\gamma p}
$$

\section{CONCLUSIONS}

For Hamilton systems, we present an approach to find Hojman conserved quantities in terms in terms of the form invariance.

\section{REFERENCES}

[1] A. E. Noether, "Invariant Variations probleme," Nachr Akad Wiss Gottingen Math. Phys. Vol. KI II, May. 1918 , pp.235-257

[2] F. X. Mei, "Lie symmetry and the Conserved Quantity of a Generalized Hamiltonian System," Acta Phys Sin, Vol. 52, May. 2003,1048-1050

[3] M. Lutzky , "Dynamical Symmetries and Conserved Quantities," Phys. A: Math Gen, vol. 12, Jun. 1979, pp.973-981

[4] S. A. Hojman, "A New Conservation Law Constructed without Using either Lagrangians or Hamiltonians," Phys.A: Math.Gen. vol. 25 , Oct. 1992, pp. L291-295

[5] F. X. Mei, "The Non-Nother conserved quantity for the Differential Equation of Motion in Phase Space," Chinese Science Bill, Vol. 47, Oct. 2002, pp.1544-1545

[6] H B Zhang, L Q Chen and S L Gu, "The General Lie Symmetries and Non-Nother conserved quantity for Birkhoff Systems," Acta. Mech. Sinica, vol. 36, Feb. 2004, pp. 254-256

[7] H. S. Lu, H. B. Zhang and S. L. Gu , " Noether's theory of Lagrange systems in discrete case," Chinese Physics B, vol. 20 , Jan. 2011, pp. 321-324

[8] S. L. Gu and H. B. Zhang, "Mei Symmetry, Noether Symmetry and Lie Symmetry of an Emden System," Acta. Phys Sin. Vol. 55, Nov. 2006, pp. 5594-5597

[9] H. B. Zhang, H. S. Lu and S. L. Gu , "The Lie Point Symmetry-Preserving Difference scheme of Holonomic Constrained Mechanical Systems," Acta. Phys Sin. Vol. 59, Aug. 2010, pp. 5213-5218

[10] H. S. Lu, H. B. Zhang and S. L. Gu , "Hojman's theorem of the third-orderordinary differential equation," Chinese Physics B, vol. 18, Aug. 2009, pp.3135-3138 